\title{
Determination of 2-Propenal Using Headspace Solid-Phase Microextraction Coupled to Gas Chromatography-Time-of-Flight Mass Spectrometry as a Marker for Authentication of Unrefined Sesame Oil
}

\author{
Ahmad Rois Mansur, ${ }^{1,2}$ Tae Gyu Nam, ${ }^{1}$ Hae Won Jang, ${ }^{1}$ Yong-Sun Cho, ${ }^{1}$ \\ Miyoung Yoo, ${ }^{1}$ Dongwon Seo, ${ }^{1}$ and Jaeho $\mathrm{Ha}^{1}$ \\ ${ }^{1}$ Korea Food Research Institute, Seongnam, Gyeonggi 13539, Republic of Korea \\ ${ }^{2}$ Department of Food Biotechnology, Korea University of Science and Technology, Daejeon 34113, Republic of Korea \\ Correspondence should be addressed to Jaeho Ha; jaeho.ha@wikim.re.kr
}

Received 27 June 2017; Accepted 25 September 2017; Published 24 October 2017

Academic Editor: Maria B. P. P. Oliveira

Copyright (c) 2017 Ahmad Rois Mansur et al. This is an open access article distributed under the Creative Commons Attribution License, which permits unrestricted use, distribution, and reproduction in any medium, provided the original work is properly cited.

\begin{abstract}
Ascertaining the authenticity of the unrefined sesame oil presents an ongoing challenge. Here, the determination of 2-propenal was performed by headspace solid-phase microextraction (HS-SPME) under mild temperature coupled to gas chromatography with time-of-flight mass spectrometry, enabling the detection of adulteration of unrefined sesame oil with refined corn or soybean oil. Employing this coupled technique, 2-propenal was detected in all tested refined corn and soybean oils but not in any of the tested unrefined sesame oil samples. Using response surface methodology, the optimum extraction temperature, equilibrium time, and extraction time for the HS-SPME analysis of 2-propenal using carboxen/polydimethylsiloxane fiber were determined to be $55^{\circ} \mathrm{C}$, $15 \mathrm{~min}$, and $15 \mathrm{~min}$, respectively, for refined corn oil and $55^{\circ} \mathrm{C}, 25 \mathrm{~min}$, and $15 \mathrm{~min}$, respectively, for refined soybean oil. Under these optimized conditions, the adulteration of unrefined sesame oil with refined corn or soybean oils (1-5\%) was successfully detected. The detection and quantification limits of 2-propenal were found to be in the range of $0.008-0.010$ and $0.023-0.031 \mu \mathrm{g} \mathrm{mL}$, respectively. The overall results demonstrate the potential of this novel method for the authentication of unrefined sesame oil.
\end{abstract}

\section{Introduction}

Unrefined sesame oil has long been considered as a popular health food due to its considerable content of bioactive compounds such as phytosterols and lignans. It is most commonly consumed as a nutritive flavor enhancer in many Asian countries [1,2]. For instance, approximately 73,000 tonnes of sesame seeds and 600 tonnes of unrefined sesame oils were imported annually in Korea during 2010-2013 to compensate for the limited domestic production of sesame seeds [3]. The retail price of unrefined sesame oil in Korea is 5 to 30 times higher than that of other refined edible oils. Therefore, the economically motivated adulteration of sesame oil, with potential impact on the oil quality, nutritional value, and safety to consumers, is a critical problem. Adulterated sesame oils are most commonly manufactured by blending pure unrefined sesame oil with cheaper and lower quality edible oils such as refined corn and soybean oils $[4,5]$.

In the past two decades, various analytical techniques combined or not with chemometric methods have been established for the verification of sesame oil authenticity. For instance, several nonseparation/nondestructive techniques (e.g., isotope ratio mass spectrometry, infrared spectroscopy, and nuclear magnetic resonance spectroscopy) have been applied for the detection of adulterated sesame oils [1, 4, 6]. Previous studies have also employed techniques such as gas chromatography (GC) coupled with a flame ionization detector [7] or mass spectrometer [8,9], high performance liquid chromatography with a refractive index detector [10], an evaporative light scattering detector $[5,11]$ or a fluorescence detector [12], an electronic nose [13], and realtime PCR [14]. Despite the recent advances in the analytical 
methods available for the detection of sesame oil adulteration, the minimum adulteration detection levels remain relatively high. Currently, it is possible to detect the presence of refined corn oil in unrefined sesame oil at $\geq 10 \%[1,4]$ and that of refined soybean oil at $\geq 3 \%$ [1]. This limitation arises mainly from the fact that sesame oil and oil adulterants (e.g., corn and soybean oils) have very similar physical, chemical, and biological properties. In addition, chemometric methods using unreliable marker compounds obtained from both sesame oil and oil adulterants might not be able to adequately detect the presence of oil adulterants in sesame oil. Additionally, the techniques described above are often time-consuming, expensive, and require the use of harmful solvents, complex technology, and expertise. Further research is therefore highly necessary in order to establish a new approach and identify specific compounds as reliable markers for the authentication of unrefined sesame oil.

Headspace solid-phase microextraction (HS-SPME) is a simple, fast, sensitive, solvent-free, and cost-efficient method for the extraction of volatile compounds in complex matrices such as those present in edible oils [15]. In addition, GC equipped with time-of-flight mass spectrometry (GCTOF/MS) allows the acquisition of full mass spectra even for volatile compounds at very low concentrations [16]. Recently, headspace coupled to GC-TOF/MS has been employed to analyze volatile compounds for the purpose of sesame oil authentication [16]. In this line, Ma et al. [15] have reported that the volatile aldehydes present in edible oils can be extracted by HS-SPME under mild temperature conditions. 2-Propenal is one of the highly reactive aldehydes detected in thermally processed edible oils, formed from the constituents of glycerides such as the glycerol moieties of triacyl and diacyl glycerides. It can also be generated by degradation of unsaturated fatty acid backbones [17]. Despite extensive studies on the pathway and mechanism of 2-propenal formation from oil constituents $[18,19]$, the determination of 2-propenal under mild temperature and its application as a detection marker of sesame oil adulteration remain vastly unexplored. The present study, therefore, aims to develop a combined approach of HS-SPME analysis under mild temperature and GC-TOF/MS for the determination of 2-propenal as a volatile marker to detect unrefined sesame oil adulteration with refined corn or soybean oil.

\section{Materials and Methods}

2.1. Materials. Twelve brands of unrefined sesame oil, six brands of refined corn oil, and five brands of refined soybean oil used in this study were manufactured by food companies in Korea. All samples were purchased from a local supermarket (Seongnam, Korea); they were all pure and qualified products. The shelf life of commercial oil samples is at least twelve months. The samples were stored in a dark and dry environment prior to analysis. To prevent any changes in chemical constituents, samples were analyzed immediately after unpacking in the laboratory. 2-Propenal was purchased from Sigma-Aldrich (St. Louis, MO, USA).

2.2. Chromatographic Conditions and Data Processing. The extracted volatiles were analyzed using a GC-TOF/MS instrument (LECO Corp., St. Joseph, MI, USA) equipped with Agilent 6890N (Agilent Technologies, Santa Clara, CA, USA). The dimensions of the primary (DB-WAX) and the secondary (DB-1701) columns were $60 \mathrm{~m} \times 0.25 \mathrm{~mm}$ internal diameter (ID) $\times 0.25 \mu \mathrm{m}$ film thickness and $20 \mathrm{~m} \times 0.10 \mathrm{~mm}$ ID $\times 0.40 \mu \mathrm{m}$ film thickness (Agilent J\&W GC Columns, USA), respectively. The temperatures of the GC inlet and transfer line were set at $220^{\circ} \mathrm{C}$. High purity helium $(99.99 \%)$ was used as the carrier gas at a constant flow rate of $1.20 \mathrm{~mL} \mathrm{~min}^{-1}$ and a split ratio of $20: 1$. The primary oven temperature was maintained at $40^{\circ} \mathrm{C}$ for $2 \mathrm{~min}$ and then ramped to $230^{\circ} \mathrm{C}$ at a rate of $20^{\circ} \mathrm{C} \mathrm{min}^{-1}$, where it was held for $10 \mathrm{~min}$. The secondary oven was always operated at a temperature $10^{\circ} \mathrm{C}$ higher than that of the primary oven throughout the analysis. The detector voltage was set to $1600 \mathrm{~V}$ with an electron energy of $70 \mathrm{eV}$. An acquisition delay of $400 \mathrm{~s}$ was employed and the mass spectra were collected in the mass range of $m / z 33-650$, at an acquisition rate of

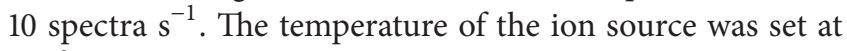
$200^{\circ} \mathrm{C}$.

The raw chromatograms were first processed using ChromaTOF $^{\circledR}$ software (LECO Corp, version 4.22) for peak finding, baseline correction, deconvolution, library matching, and area calculation. The peaks were extracted with a signal-to-noise ratio $(\mathrm{S} / \mathrm{N})$ of 20 . The peaks were identified tentatively by comparison of the obtained mass spectra with those available in mass spectral databases (NIST and Wiley), with a minimum similarity match of 800 . The area of each peak was calculated using the unique mass of each detected volatile compound. After the initial processing, all the peak information from different chromatograms was aligned using the Statistical Compare (SC) option in the ChromaTOF software in order to obtain an accurate comparison of the volatile profiles of sesame oil and oil adulterants (corn and soybean oils). Following the alignment process, the basic statistical information from different peak calculations was compared in order to identify the volatile compounds with potentially significant class differences between volatiles from sesame oil and the oil adulterants. This comparison was necessary in order to identify compounds that could be used as potential markers for the detection of sesame oil adulteration. The results of the SC process were exported as .csv files and used for further analysis.

\subsection{Preliminary Experiments}

2.3.1. Selection of Volatile Markers for Authentication of Sesame Oil. The initial step of the preliminary experiment involved the selection of volatile marker compounds for the detection of sesame oil adulteration. Commercial manual sampling SPME devices and $85 \mu \mathrm{m}$ carboxen/polydimethylsiloxane (CAR/PDMS) fiber (Supelco, PA, USA) were selected for the extraction of volatile compounds in oil samples. Oil samples (each $2 \mathrm{~g}$ ) were heated at $55^{\circ} \mathrm{C}$ for $15 \mathrm{~min}$ (equilibrium time) in a $20 \mathrm{~mL}$ glass vial equipped with a PTFE/silicone septum (Supelco, PA, USA) and extracted using a CAR/PDMS fiber for $15 \mathrm{~min}$. After extraction, the SPME fiber was immediately thermally desorbed in the injection port of the GC-TOF/MS. Next, the volatile compounds present in refined corn and 
TABLE 1: Experimental factors and their coded and actual values used for HS-SPME optimization using response surface methodology.

\begin{tabular}{lcccc}
\hline \multirow{2}{*}{ Factor } & \multirow{2}{*}{ Symbol } & \multicolumn{3}{c}{ Coded levels } \\
& & -1 & 0 & +1 \\
\hline Extraction temperature $\left({ }^{\circ} \mathrm{C}\right)$ & $X_{1}$ & 25 & 40 & 55 \\
Equilibrium time $(\mathrm{min})$ & $X_{2}$ & 15 & 30 & 45 \\
Extraction time $(\mathrm{min})$ & $X_{3}$ & 5 & 10 & 15 \\
\hline
\end{tabular}

soybean oils but not in unrefined sesame oils were selected as adulteration detection markers. Among these compounds, 2-propenal was selected and used for the optimization of the HS-SPME conditions.

2.3.2. Fiber Selection for the Extraction of 2-Propenal. Four SPME fibers, (i) $85 \mu \mathrm{m} \mathrm{CAR/PDMS,} \mathrm{(ii)} 50 / 30 \mu \mathrm{m}$ divinylbenzene/CAR/PDMS (DVB/CAR/PDMS), (iii) $65 \mu \mathrm{m}$ PDMS/DVB, and (iv) $100 \mu \mathrm{m}$ PDMS, were evaluated to select the most suitable fiber for the extraction of 2-propenal in oil samples. A sample of corn or soybean oil (2g) was heated at $55^{\circ} \mathrm{C}$ for $15 \mathrm{~min}$ (equilibrium time) in a glass vial, extracted using the four selected SPME fibers for $15 \mathrm{~min}$, and then thermally desorbed in the GC-TOF/MS. The adsorption efficiencies on the SPME fibers were evaluated by comparison of the peak areas of 2-propenal.

2.3.3. Temperature Selection for the Extraction of 2-Propenal. The final step in the preliminary set of experiments was the determination of the most appropriate temperature for the extraction of 2-propenal. The extraction temperature was varied systematically from 25 to $100^{\circ} \mathrm{C}$, while the equilibrium and extraction times were both held constant at $15 \mathrm{~min}$. In the present study, CAR/PDMS and DVB/CAR/PDMS fibers were chosen because of their higher extraction efficiencies compared to those of the PDMS/DVB and PDMS fibers. Under different extraction temperatures, the 2-propenal in corn or soybean oils (each $2 \mathrm{~g}$ ) was extracted using CAR/PDMS or $\mathrm{DVB} / \mathrm{CAR} / \mathrm{PDMS}$ fiber. Based on the obtained results, the CAR/PDMS fiber and extraction temperatures of $25-55^{\circ} \mathrm{C}$ were selected for the optimization of the HS-SPME analysis using response surface methodology (RSM).

2.4. Optimization of HS-SPME Conditions Using RSM. The effect of three different SPME factors including the extraction temperature $\left(X_{1}, 25-55^{\circ} \mathrm{C}\right)$, equilibrium time $\left(X_{2}, 15-45 \mathrm{~min}\right)$, and extraction time $\left(X_{3}, 5-15 \mathrm{~min}\right)$ was evaluated by RSM (Table 1). The analytical design consisted of 34 experimental points in a randomized order, and six replicates were performed for the center point and used for the estimation of the pure error sum of squares (Supp. Table 1 in Supplementary Material available online at https://doi.org/ 10.1155/2017/9106409). The experimental points were divided into two blocks, each consisting of 17 experiments. 2Propenal in corn or soybean oils (each $2 \mathrm{~g}$ ) was extracted using CAR/PDMS fiber under the extraction conditions based on the design described in Supp. Table 1 in Supplementary Material. The obtained experimental data were then fitted to the following nonlinear polynomial equation:

$$
Y=\beta_{0}+\Sigma \beta_{i} X_{i}+\Sigma \beta_{i i} X_{i}^{2}+\Sigma \beta_{i j} X_{i} X_{j}
$$

where $Y$ is the response value predicted by the model; $\beta_{0}$ is a constant; and $\beta_{i}, \beta_{i i}$, and $\beta_{i j}$ are the linear, quadratic, and interaction coefficients, respectively. The adequacy of the predicted model was evaluated using modeling analysis, coefficients of determination $\left(R^{2}\right)$, and lack-of-fit tests.

\subsection{Determination of 2-Propenal in Sesame Oils Adulterated} with Corn or Soybean Oils. Samples of adulterated sesame oil were prepared shortly before analysis by randomly blending unrefined sesame oils with refined corn or soybean oils. The concentrations of refined corn or soybean oils in the adulterated sesame oil were 1,5 , and $10 \%(\mathrm{w} / \mathrm{w})$. Three corn oils and three soybean oils were used as refined adulterants and 18 admixtures were prepared. Each oil sample was analyzed quantitatively in triplicate. 2-Propenal in the pure and adulterated oils was extracted using HS-SPME under the optimal conditions determined by RSM.

A stock solution of 2-propenal $\left(750 \mathrm{mg} \mathrm{L}^{-1}\right)$ was prepared in absolute ethanol (Burdick \& Jackson, MI, USA) [20] and stored at $4^{\circ} \mathrm{C}$ prior to analysis. The stock solution was subsequently diluted with absolute ethanol to produce intermediate standard solutions at concentrations ranging from 0.02 to $0.76 \mu \mathrm{g} \mathrm{mL}^{-1}$. Each standard solution was prepared shortly before exposure to the CAR/PDMS fiber. Each standard solution $(2 \mathrm{~g})$ was extracted and injected in the GC-TOF/MS (six replicates) in order to obtain calibration curves. The average peak areas determined from the analysis of each of these standard solutions were used to quantify the concentration of 2-propenal in the oil samples and determine the limits of detection (LOD) and limits of quantification (LOQ).

2.6. Statistical Analysis. The response surface modeling was performed using the Minitab statistical package (Minitab Inc., USA, version 17). The differences between the treatments employed in the preliminary experiments were determined using one-way analysis of variance (ANOVA) followed by Tukey's test $(p<0.05)$. A two-sample $t$-test was performed in order to establish the differences between the predicted and observed peak areas of 2-propenal in corn and soybean oils $(p<0.05)$. These two statistical tests were conducted using the IBM SPSS Statistics software (SPSS Inc., USA, version 20).

\section{Results and Discussion}

3.1. Preliminary Experiments. The volatile compounds in the tested sesame, corn, and soybean oils were extracted using CAR/PDMS fiber and analyzed by GC-TOF/MS. The volatile compounds found only in corn and soybean oils were then selected as markers for the detection of sesame oil adulteration. Among the several marker compounds, 2propenal was selected as an adulteration marker owing to its high peak area and reproducibility (data not shown). 2Propenal was not found in all the tested unrefined sesame 


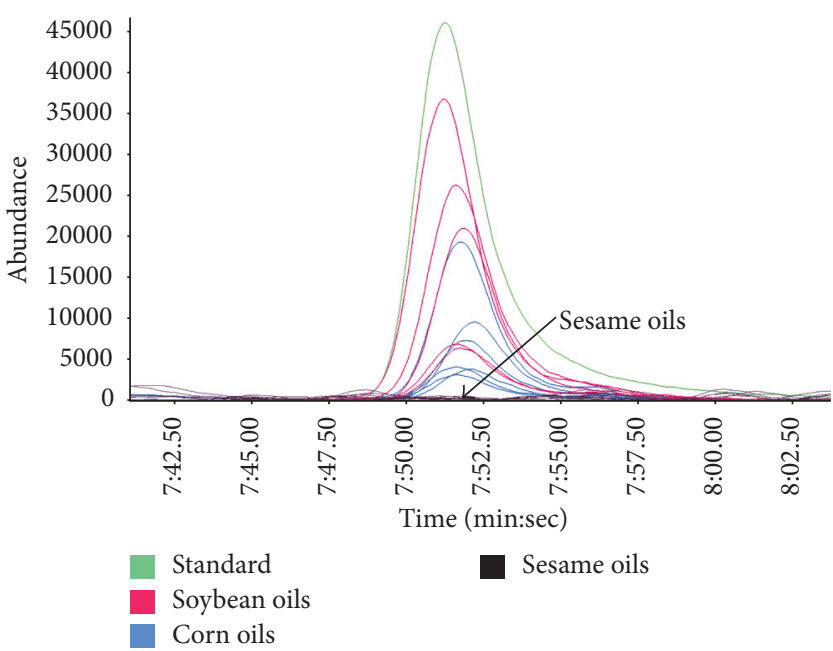

(a)

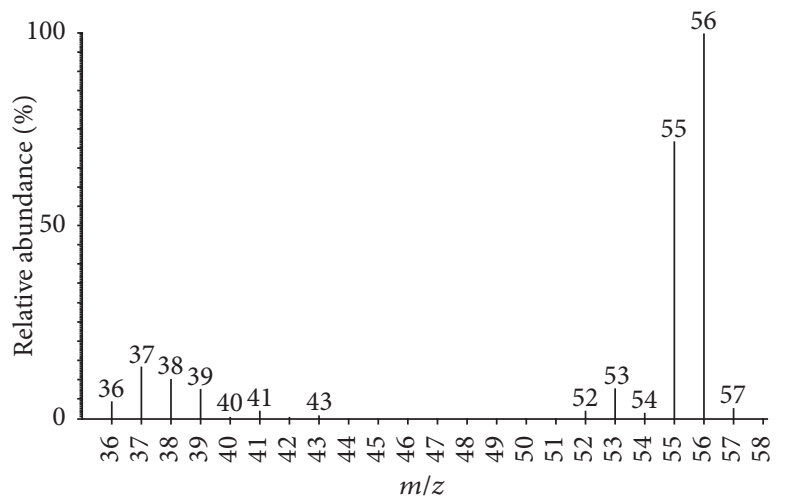

(c)

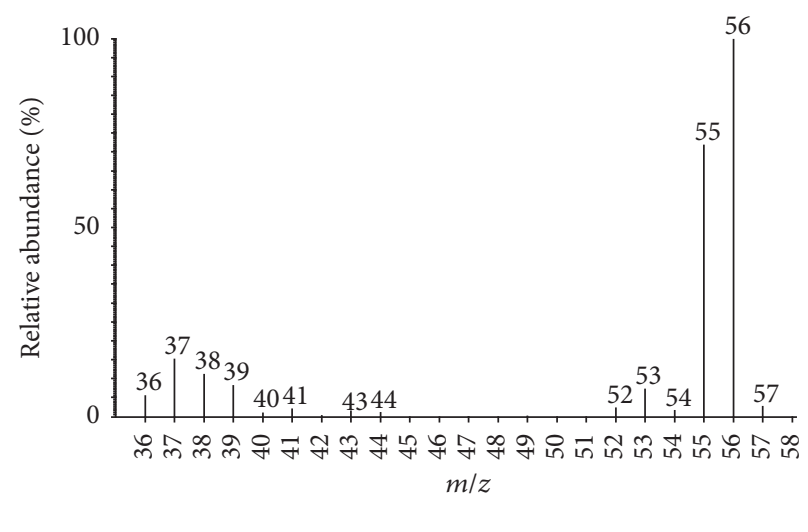

(b)

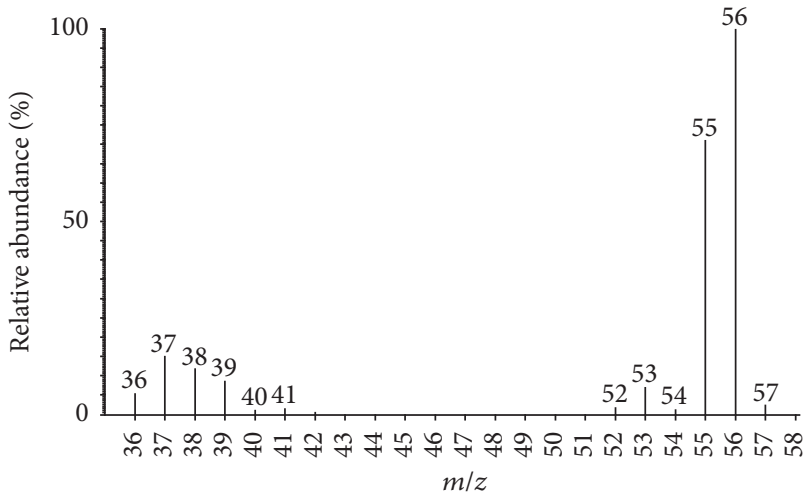

(d)

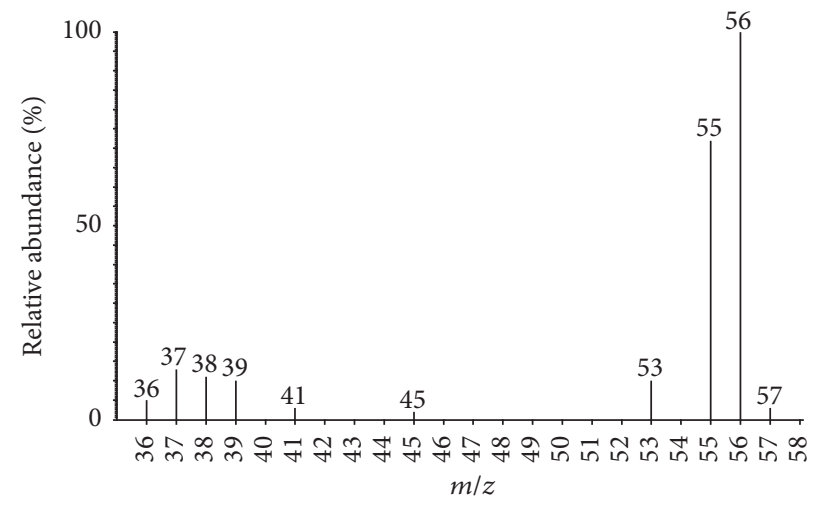

(e)

Figure 1: Chromatograms of 2-propenal in six corn oils (blue line), five soybean oils (red line), and twelve sesame oils (black line) analyzed using GC-TOF/MS (a). Similar MS spectra of 2-propenal in corn oil (b), soybean oil (c), authentic standard (d), and Wiley library (e) were detected at $70 \mathrm{eV}$ of electron energy.

oils, while it was found in all the tested refined corn and soybean oils (Figure 1(a)). The presence of 2-propenal in corn and soybean oils (Figures 1(a), 1(b), and 1(c)) was confirmed by comparing the results to the retention time and mass spectra of standard solution containing 2-propenal at known concentrations (Figure $1(\mathrm{~d})$ ), as well as by comparison with a spectral library (Figure 1(e)). The mass spectrum (molecular ion at $m / z 56$ and fragment ions at $m / z 37,38,39,53$, and 55) of 2-propenal was obtained by the electron ionization mass spectrometry.

In order to determine the most suitable fiber to extract 2-propenal from corn and soybean oils, four SPME fibers (CAR/PDMS, DVB/CAR/PDMS, PDMS/DVB, and PDMS) were evaluated. Selection of the appropriate fiber coating is 


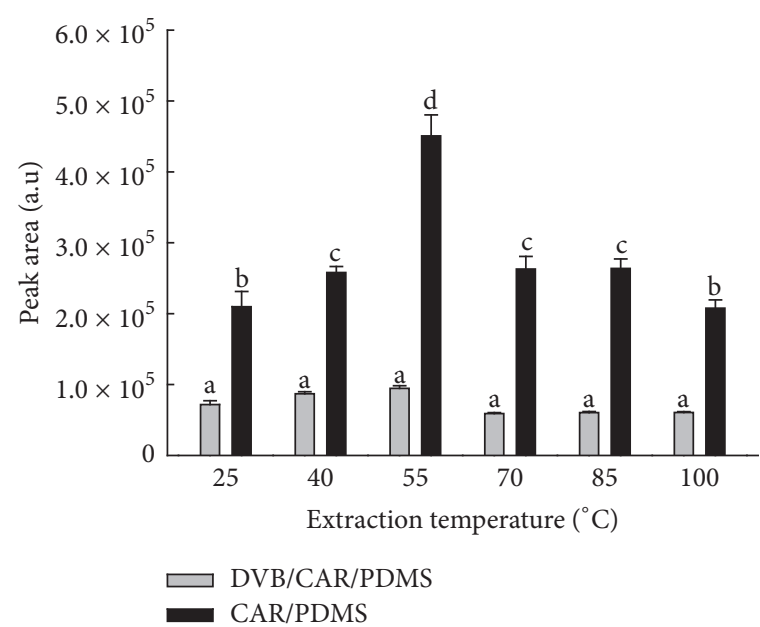

(a)

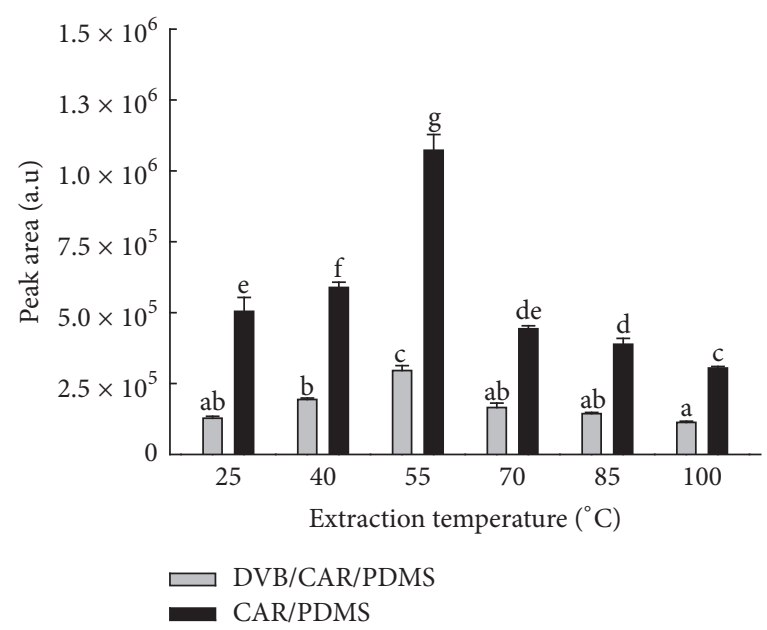

(b)

Figure 2: Comparison of DVB/CAR/PDMS and CAR/PDMS fibers on the extraction efficiencies of 2-propenal from corn (a) and soybean (b) oils at different temperatures $\left(25-100^{\circ} \mathrm{C}\right)$. Different letters indicate the significant differences at $p<0.05$.

one of the most critical steps in the optimization of SPME method. The suitability of a particular fiber coating for the analyte of interest is related directly to the properties of the extraction phase, which in turn determine the overall selectivity and reliability of the method [21]. The extraction efficiency of the SPME fibers was determined by comparing the peak areas of 2-propenal. PDMS and PDMS/DVB fibers were excluded from further analysis after a preliminary set of experiments. These two fibers had much lower extraction efficiencies compared to those of the CAR/PDMS and DVB/CAR/PDMS fibers (data not shown), indicating that the polarity of these two fibers makes them unsuitable for the extraction of 2-propenal in oil samples.

The performance of CAR/PDMS and DVB/CAR/PDMS fibers in the extraction of 2-propenal from oil samples was then further investigated and compared under different extraction temperatures $\left(25-100^{\circ} \mathrm{C}\right)$. Results in Figure 2 show that the use of CAR/PDMS fiber resulted in a higher 2propenal peak area $(p<0.05)$ for both corn and soybean oils compared to that determined using DVB/CAR/PDMS fiber at all extraction temperatures. The peak areas of 2-propenal in both corn and soybean oils extracted with CAR/PDMS fiber at $55^{\circ} \mathrm{C}$ were significantly higher $(p<0.05)$ than those obtained using the same fiber at other extraction temperatures. The peak areas of 2-propenal in corn and soybean oils extracted using CAR/PDMS fiber were found to decrease markedly at temperatures above $70^{\circ} \mathrm{C}$. Generally, heating increases the vapor pressure and enhances mass transfer processes, thereby facilitating the release of analytes into the headspace. Nonetheless, excessive heating can have an adverse effect on the adsorption of analytes by the fiber coating as a result of reduction of the distribution constant [22]. Accordingly, CAR/PDMS fiber and extraction temperatures of $25-55^{\circ} \mathrm{C}$ were chosen as the starting point for further optimization of the HS-SPME conditions for the extraction of 2-propenal from refined corn and soybean oils using RSM.

3.2. Optimization of HS-SPME Using RSM. The mechanism of HS-SPME is based on the equilibrium distribution of analytes between three phases (fiber coating, headspace, and sample) and, therefore, the vapor pressure of the volatile analytes in the vial can affect significantly the extraction of volatile analytes present in the headspace by SPME. The main SPME variables/factors affecting the vapor pressure and equilibrium state of volatile compounds in the headspace are the extraction temperature, equilibrium time, and extraction time [22]. The drawback of the preliminary experiments described above, in which the effect of one SPME variable/factor (the extraction temperature) was evaluated while the remaining parameters (e.g., equilibrium and extraction time) remained constant, is that the optimization of a single variable is unlikely to produce the most optimal conditions for the extraction of 2-propenal. In contrast, RSM enables the adequate evaluation of the relative influence of all the experimental variables during the optimization process, even in the presence of complex interaction relationships [23]. RSM employs an initial modeling step, followed by the determination of the optimal parameter ranges. The modeling process is performed by adjusting first- or second-order polynomial equations to the data obtained experimentally, followed by the ANOVA.

In this study, the experimental response $(Y$, peak area of the 2-propenal) was modeled as a function of linear, quadratic, and interaction effects of the extraction temperature $\left(X_{1}\right)$, equilibrium time $\left(X_{2}\right)$, and extraction time $\left(X_{3}\right)$. The results show that the predicted quadratic polynomial models provide a good fit to the experimental data, exhibiting relatively high values for the coefficient of determination $\left(R^{2}\right)$ of 0.83 and 0.98 for corn and soybean oils, respectively (Table 2). In general, the closer the $R^{2}$ value is to unity, the better the fit between the theoretical model and experimental data is. The results of the ANOVA show that both the linear and quadratic parameters were not significant $(p>0.05)$ in corn oil (Table 3). Similarly, interactions between the parameters, except for the interaction between the extraction temperature and equilibrium time, also did not produce a significant effect $(p>0.05)$. Thus, the interplay between the 


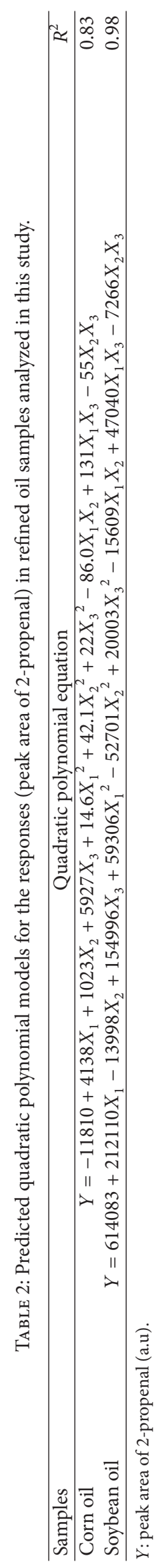




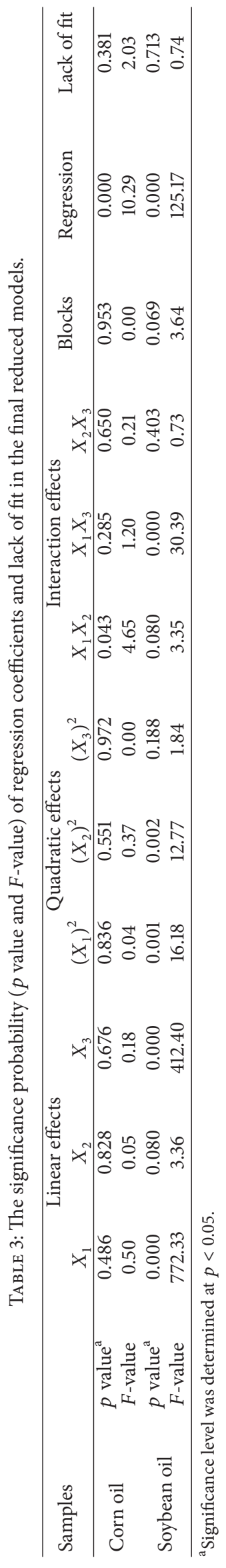




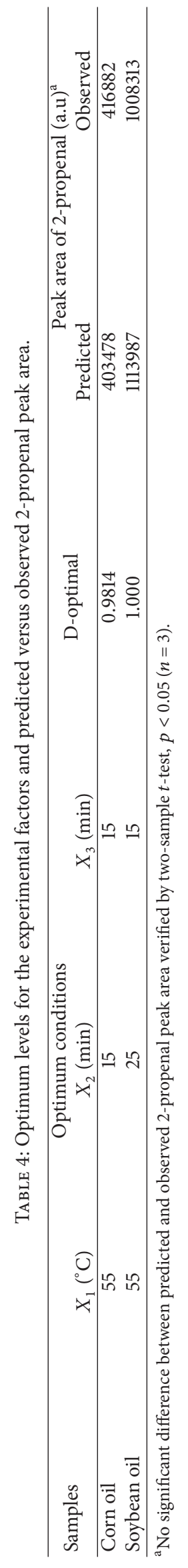


TABLE 5: Concentrations of 2-propenal in corn, soybean, sesame, and adulterated sesame oils.

\begin{tabular}{|c|c|c|c|c|c|}
\hline \multirow{2}{*}{ Oil adulterants ${ }^{\mathrm{a}}$} & \multicolumn{5}{|c|}{ 2-Propenal $\left(\mu \mathrm{gg}^{-1}\right.$ oil $)$} \\
\hline & $100: 0^{\mathrm{b}}$ & $10: 90$ & $5: 95$ & $1: 99$ & $0: 100$ \\
\hline CO 1:SSO & $1.302 \pm 0.085^{c}$ & $0.115 \pm 0.004$ & $0.084 \pm 0.005$ & $0.062 \pm 0.003$ & $\mathrm{ND}^{\mathrm{d}}$ \\
\hline $\mathrm{CO} 2$ : SSO & $0.304 \pm 0.021$ & $0.037 \pm 0.002$ & $0.025 \pm 0.001$ & ND & ND \\
\hline $\mathrm{CO} 3$ : SSO & $0.578 \pm 0.024$ & $0.075 \pm 0.008$ & $0.056 \pm 0.007$ & $0.031 \pm 0.002$ & ND \\
\hline SBO 1: SSO & $3.557 \pm 0.040$ & $0.085 \pm 0.007$ & $0.057 \pm 0.003$ & $0.018 \pm 0.004$ & ND \\
\hline SBO 2: SSO & $2.630 \pm 0.030$ & $0.073 \pm 0.006$ & $0.035 \pm 0.007$ & $\mathrm{ND}$ & ND \\
\hline SBO 3 : SSO & $0.609 \pm 0.030$ & $0.025 \pm 0.003$ & $0.008 \pm 0.001$ & $\mathrm{ND}$ & $\mathrm{ND}$ \\
\hline
\end{tabular}

${ }^{a}$ Oil adulterants consisted of three different brands of corn or soybean oils; each oil adulterant was analyzed quantitatively in triplicate. ${ }^{\mathrm{b}}$ Corn or soybean oil/sesame oil $(\mathrm{w} / \mathrm{w}) .{ }^{\mathrm{c}}$ All data are presented as mean \pm standard deviation $(n=3) ;{ }^{\mathrm{d}}$ not detected; CO: corn oil; SBO: soybean oil; SSO: sesame oil.

extraction temperature and equilibrium time was established as the primary determining term that may cause significant $(p<0.05)$ changes in the observed response (peak area of 2propenal). For soybean oil, on the other hand, both the linear (extraction temperature and extraction time) and quadratic (extraction temperature and equilibrium time) parameters were found to play a significant role $(p<0.05)$ influencing the total peak area of the 2-propenal. Further, the interaction between the extraction temperature and extraction time was also found to be significant $(p<0.05)$. The predicted models provided an excellent fit to the experimental data for both oil samples examined in this study, with a regression of $p<$ 0.05 and an insignificant $(p>0.05)$ lack of fit. In addition, no significant differences $(p>0.05)$ were found between the two experimental blocks, demonstrating that there were no significant differences between measurements obtained at different experimental periods.

Table 4 shows the optimum values for the independent HS-SPME variables/factors obtained using the "response optimizer" option available in the Minitab statistical package, which afforded composite desirability values of 0.98 and 1.00 for corn and soybean oils, respectively. In order to validate the proposed model, triplicate experiments were conducted under the optimum experimental conditions and the results were compared to those obtained by predictions. The validation results revealed that the experimental values of the 2propenal peak area were not significantly different $(p<0.05)$ from the predicted values, thereby confirming the validity and adequacy of the predictive models. The optimum HSSPME extraction temperature, equilibrium time, and extraction time for corn and soybean oils were determined to be $55^{\circ} \mathrm{C}, 15 \mathrm{~min}, 15 \mathrm{~min}$, and $55^{\circ} \mathrm{C}, 25 \mathrm{~min}, 15 \mathrm{~min}$, respectively.

\subsection{Application of 2-Propenal as a Detection Marker of Sesame} Oil Adulteration. To date, there is no consensus in the literature as to the origin of 2-propenal in edible oils. In this study, we confirmed that 2-propenal was not found in the tested unrefined sesame oil samples equilibrated and extracted at $55^{\circ} \mathrm{C}$ (Figure 1(a)), while it was detected in all the tested refined corn and soybean oils even at room temperature (Figure 2). In a previous study, 2-propenal was produced via thermal oxidation of vegetable oils at temperatures above $100^{\circ} \mathrm{C}$ [18]. This finding may be attributed to the thermal oxidation of refined corn and soybean oils during industrial refining stages because of the high temperatures $\left(\geq 200^{\circ} \mathrm{C}\right)$ used. As shown in Supp. Figure 1 in Supplementary Material, thermal oxidation of unrefined sesame oil at $150^{\circ} \mathrm{C}$ resulted in the formation of 2-propenal. However, pressing sesame seeds without refining is a common process for the production of sesame oil in Asia. A possible reason is that the process for unrefined sesame oil production does not involve a refining stage using the high temperatures; therefore, 2-propenal may not be formed during sesame oil production.

The formation of lipid oxidation products including 2propenal in refined corn and soybean oils may also be due to the lack of considerable amounts of antioxidants. The refining process for edible oil results in a huge loss of antioxidants such as $\alpha$-tocopherol due to the high operating temperatures [24]. Suja et al. [25] have reported that the addition of antioxidants in edible oils such as soybean and sunflower oils is effective in the inhibition of the lipid oxidation. Unrefined sesame oil, on the other hand, possesses high contents of endogenous antioxidants such as sesamolin, sesamin, sesamol, and phenolic compounds [26, 27]. In addition, sesame seed roasting prior to oil extraction can improve significantly the oxidative stability of the obtained oils as a result of the antioxidants produced via Maillard reactions. The oxidative stability is known to be positively correlated with the increasing roasting temperature of sesame seeds [28]. The natural antioxidants in unrefined sesame oil are likely able to inhibit the lipid oxidation, preventing the formation of 2-propenal.

In this study, unrefined sesame oil samples were randomly adulterated with corn or soybean oil samples at concentrations of 1,5 , and $10 \%(\mathrm{w} / \mathrm{w})$. The authentic and adulterated sesame oils were extracted using HS-SPME under the optimum conditions described in Table 4 and analyzed by GC-TOF/MS. The concentration of 2-propenal in the adulterated sesame oils was then determined in order to investigate the applicability of 2-propenal as a marker for the detection of sesame oil adulteration. The results show that the concentration of 2-propenal was found to increase gradually with the increasing content of added corn or soybean oils (Table 5). 2-Propenal in sesame oil samples adulterated with refined corn or soybean oils was detected even at the lowest concentration of $1 \%$. According to the results of the admixtures presented in Table 5, 2-propenal can be used as a marker for the discrimination of unrefined sesame oil adulteration with the refined oils tested in this work.

The analytical parameters for the evaluation of the proposed method are shown in Table 6 . The linear range 


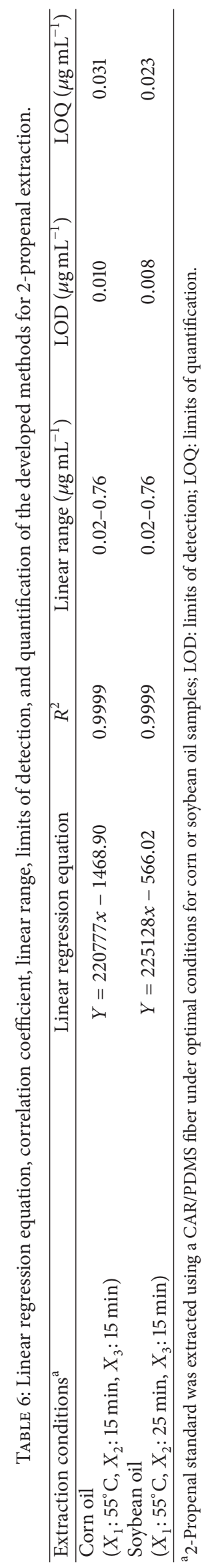


covers from 0.02 to $0.76 \mu \mathrm{g} \mathrm{mL}^{-1}$ for the 2-propenal authentic standard extracted using HS-SPME under optimal conditions for corn or soybean oil samples, and all the correlation coefficients were $>0.99$. The LOD and LOQ were found in the range of $0.008-0.010$ and $0.023-0.031 \mu \mathrm{g} \mathrm{mL}^{-1}$, respectively. Overall, the results confirm that the developed method can adequately detect the presence of refined corn and soybean oils at concentrations between 1 and 5\% (w/w) in adulterated sesame oil.

\section{Conclusion}

The present work demonstrates an optimized HS-SPME method coupled to GC-TOF/MS for the effective extraction and detection of 2-propenal in refined corn and soybean oils. The application of 2-propenal as a marker allowed the detection of unrefined sesame oil adulteration with refined corn or soybean oil even at very low adulteration levels of $1-5 \%(w / w)$. In future work, this developed method will be further tested for the detection of unrefined sesame oil adulteration with other potential oil adulterants such as refined canola and rapeseed oils.

\section{Disclosure}

Jaeho Ha's present address is World Institute of Kimchi, Gwangju 61755, Republic of Korea.

\section{Conflicts of Interest}

The authors declare that there are no conflicts of interest associated with this publication.

\section{Authors' Contributions}

Ahmad Rois Mansur and Tae Gyu Nam contributed equally to this work.

\section{Acknowledgments}

This work was supported by Main Research Program (E0152202-02, 2016) of Korea Food Research Institute funded by the Ministry of Science, ICT \& Future Planning, Republic of Korea. The authors are grateful to Mr. Byeong Ho Kim (LECO Korea CO., LTD) for his expert technical assistance.

\section{References}

[1] J. Kim, G. Jin, Y. Lee, H. S. Chun, S. Ahn, and B. H. Kim, "Combined analysis of stable isotope, $1 \mathrm{H}$ NMR, and fatty acid to verify sesame oil authenticity," Journal of Agricultural and Food Chemistry, vol. 63, no. 40, pp. 8955-8965, 2015.

[2] W.-H. Wu, "The contents of lignans in commercial sesame oils of Taiwan and their changes during heating," Food Chemistry, vol. 104, no. 1, pp. 341-344, 2007.

[3] Food and Agriculture Organization of the United Nations, "Crops and livestock products," http://faostat.fao.org/beta/en/ \#data/TP, 2016.

[4] H.-Y. Seo, J. Ha, D.-B. Shin et al., "Detection of corn oil in adulterated sesame oil by chromatography and carbon isotope analysis," Journal of the American Oil Chemists' Society, vol. 87, no. 6, pp. 621-626, 2010.

[5] Y. W. Park, P.-S. Chang, and J. Lee, "Application of triacylglycerol and fatty acid analyses to discriminate blended sesame oil with soybean oil," Food Chemistry, vol. 123, no. 2, pp. 377-383, 2010.

[6] L. Xu, Z.-H. Ye, S.-M. Yan et al., "Combining local wavelength information and ensemble learning to enhance the specificity of class modeling techniques: identification of food geographical origins and adulteration," Analytica Chimica Acta, vol. 754, pp. 31-38, 2012.

[7] D. Peng, Y. Bi, X. Ren, G. Yang, S. Sun, and X. Wang, "Detection and quantification of adulteration of sesame oils with vegetable oils using gas chromatography and multivariate data analysis," Food Chemistry, vol. 188, pp. 415-421, 2015.

[8] F.-F. Ai, J. Bin, Z.-M. Zhang et al., "Application of random forests to select premium quality vegetable oils by their fatty acid composition," Food Chemistry, vol. 143, pp. 472-478, 2014.

[9] L. Zhang, P. Li, X. Sun et al., "Classification and adulteration detection of vegetable oils based on fatty acid profiles," Journal of Agricultural and Food Chemistry, vol. 62, no. 34, pp. 87458751, 2014.

[10] D.-S. Lee, E.-S. Lee, H.-J. Kim, S.-O. Kim, and K. Kim, "Reversed phase liquid chromatographic determination of triacylglycerol composition in sesame oils and the chemometric detection of adulteration," Analytica Chimica Acta, vol. 429, no. 2, pp. 321$330,2001$.

[11] W.-J. Lee, N.-W. Su, M.-H. Lee, and J.-T. Lin, "Assessment of authenticity of sesame oil by modified Villavecchia Test and HPLC-ELSD analysis of triacylglycerol profile," Food Research International, vol. 53, no. 1, pp. 195-202, 2013.

[12] S. Aued-Pimentel, E. Takemoto, R. Antoniassi, and E. S. G. Badolato, "Composition of tocopherols in sesame seed oil: An indicative of adulteration," Grasas y Aceites, vol. 57, no. 2, pp. 205-210, 2006.

[13] Z. Hai and J. Wang, "Detection of adulteration in camellia seed oil and sesame oil using an electronic nose," European Journal of Lipid Science and Technology, vol. 108, no. 2, pp. 116-124, 2006.

[14] J. He, W. Xu, Y. Shang et al., "Development and optimization of an efficient method to detect the authenticity of edible oils," Food Control, vol. 31, no. 1, pp. 71-79, 2013.

[15] C. Ma, J. Ji, C. Tan et al., "Headspace solid-phase microextraction coupled to gas chromatography for the analysis of aldehydes in edible oils," Talanta, vol. 120, pp. 94-99, 2014.

[16] F. Zhao, J. Liu, X. Wang, P. Li, W. Zhang, and Q. Zhang, "Detection of adulteration of sesame and peanut oils via volatiles by $\mathrm{GC} \times \mathrm{GC}-\mathrm{TOF} / \mathrm{MS}$ coupled with principal components analysis and cluster analysis," European Journal of Lipid Science and Technology, vol. 115, no. 3, pp. 337-347, 2013.

[17] J. F. Stevens and C. S. Maier, "Acrolein: Sources, metabolism, and biomolecular interactions relevant to human health and disease," Molecular Nutrition \& Food Research, vol. 52, no. 1, pp. 7-25, 2008.

[18] A. Ewert, M. Granvogl, and P. Schieberle, "Isotope-labeling studies on the formation pathway of acrolein during heat processing of oils," Journal of Agricultural and Food Chemistry, vol. 62, no. 33, pp. 8524-8529, 2014.

[19] A. Shibata, M. Uemura, M. Hosokawa, and K. Miyashita, "Formation of acrolein in the autoxidation of triacylglycerols with different fatty acid compositions," Journal of the American Oil Chemists' Society, vol. 92, no. 11-12, pp. 1661-1670, 2015. 
[20] M. Kächele, Y. B. Monakhova, T. Kuballa, and D. W. Lachenmeier, "NMR investigation of acrolein stability in hydroalcoholic solution as a foundation for the valid HS-SPME/GCMS quantification of the unsaturated aldehyde in beverages," Analytica Chimica Acta, vol. 820, pp. 112-118, 2014.

[21] G. Ouyang, D. Vuckovic, and J. Pawliszyn, "Nondestructive sampling of living systems using in vivo solid-phase microextraction," Chemical Reviews, vol. 111, no. 4, pp. 2784-2814, 2011.

[22] A. Spietelun, A. Kloskowski, W. Chrzanowski, and J. Namieśnik, "Understanding solid-phase microextraction: Key factors influencing the extraction process and trends in improving the technique," Chemical Reviews, vol. 113, no. 3, pp. 1667-1685, 2013.

[23] M. A. Bezerra, R. E. Santelli, E. P. Oliveira, L. S. Villar, and L. A. Escaleira, "Response surface methodology (RSM) as a tool for optimization in analytical chemistry," Talanta, vol. 76, no. 5, pp. 965-977, 2008.

[24] J. Wei, L. Chen, X. Qiu et al., "Optimizing refining temperatures to reduce the loss of essential fatty acids and bioactive compounds in tea seed oil," Food and Bioproducts Processing, vol. 94, pp. 136-146, 2015.

[25] K. P. Suja, J. T. Abraham, S. N. Thamizh, A. Jayalekshmy, and C. Arumughan, "Antioxidant efficacy of sesame cake extract in vegetable oil protection," Food Chemistry, vol. 84, no. 3, pp. 393400, 2004.

[26] Z. Konsoula and M. Liakopoulou-Kyriakides, "Effect of endogenous antioxidants of sesame seeds and sesame oil to the thermal stability of edible vegetable oils," LWT-Food Science and Technology, vol. 43, no. 9, pp. 1379-1386, 2010.

[27] S. W. Lee, M. K. Jeung, M. H. Park, S. Y. Lee, and J. Lee, "Effects of roasting conditions of sesame seeds on the oxidative stability of pressed oil during thermal oxidation," Food Chemistry, vol. 118, no. 3, pp. 681-685, 2010.

[28] E. Choe and D. B. Min, "Mechanisms and factors for edible oil oxidation," Comprehensive Reviews in Food Science and Food Safety, vol. 5, no. 4, pp. 169-186, 2006. 

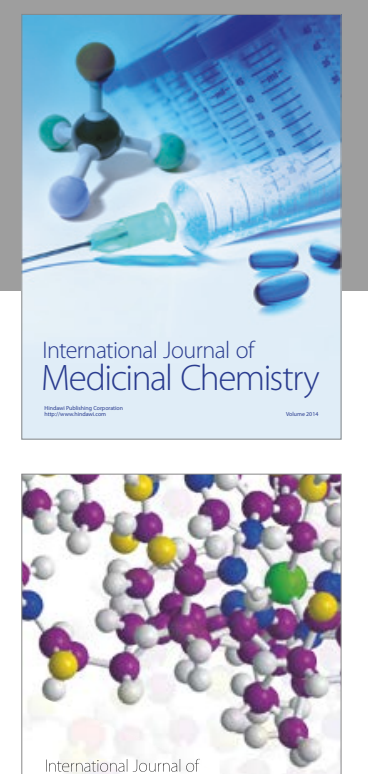

Carbohydrate Chemistry

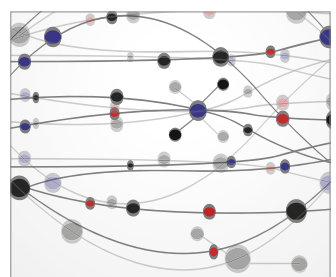

The Scientific World Journal
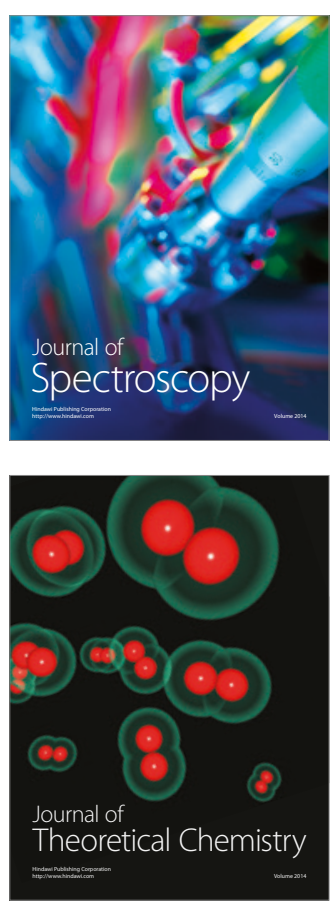
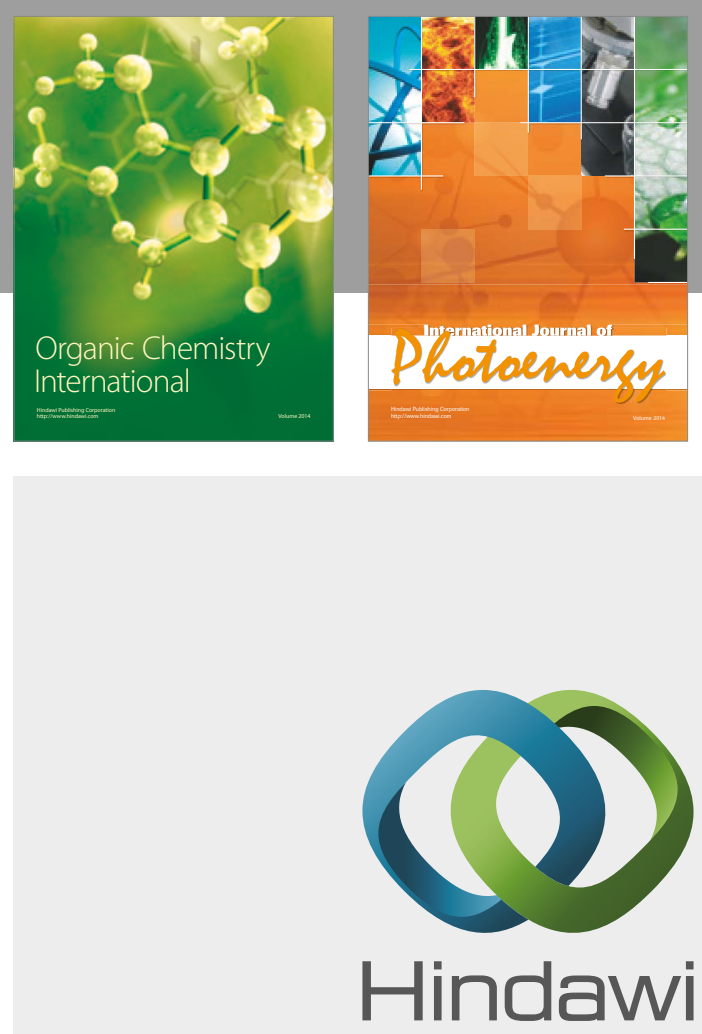

Submit your manuscripts at

https://www.hindawi.com

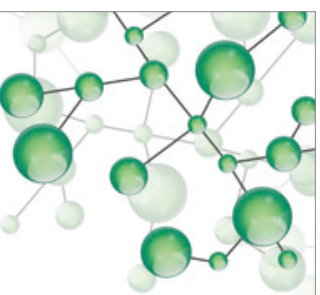

International Journal of

Inorganic Chemistry

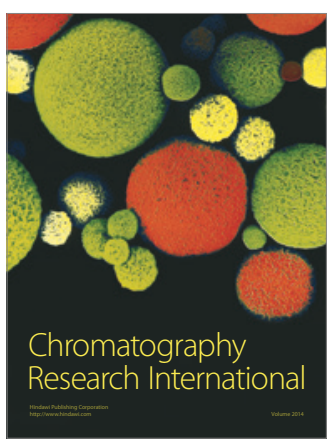

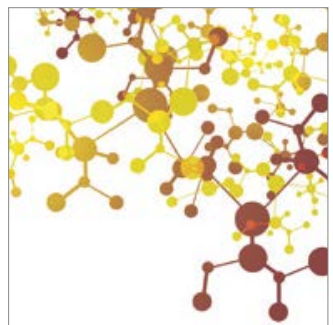

Applied Chemistry
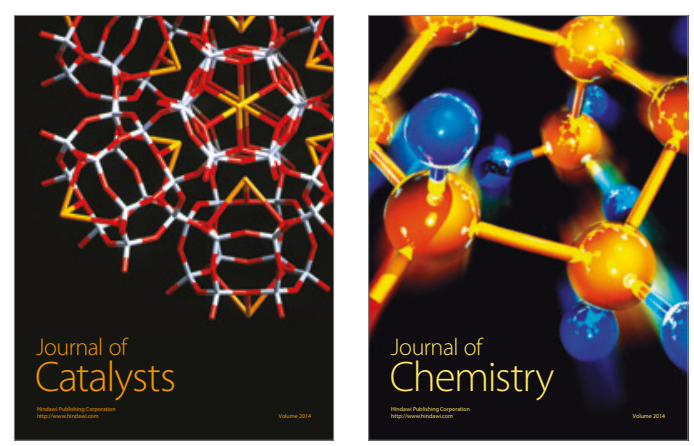
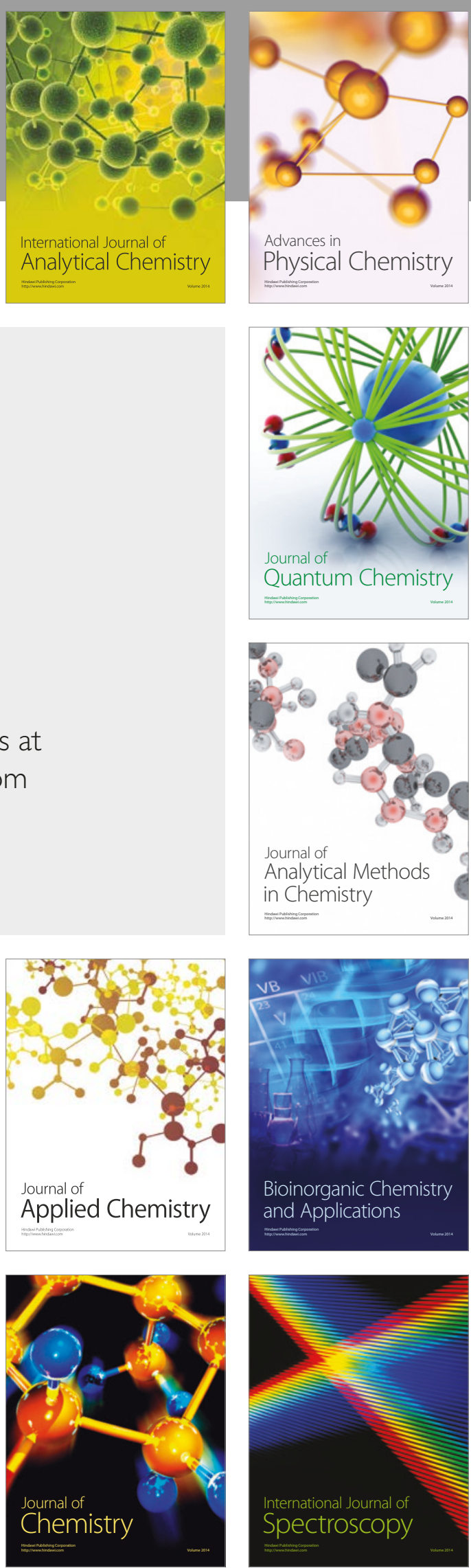